\title{
Nonlinear Switching Transient Field Simulation of Cable Joint without Residual Charge
}

\author{
Mingyan Wu1', Jun Xiong1, Lei Liao', Lu Zhu1, Ruxin Zhang1, Zheng Wu1, Gang Du1, \\ Xueyou Huang1, Haiming Li², Jian Zhang2, Sizhuo Liao², Binxian Lu${ }^{2 *}$ \\ ${ }^{1}$ Guagnzhou Power Supply CSG, Guangzhou, China \\ ${ }^{2}$ Department of Electrical and Electronic Engineering, North China Electric Power University, Beijing, China \\ Email: 254689291@qq.com, 404127726@qq.com, 33859515@qq.com, 315995738@qq.com, vanishangel@163.com, \\ 469298598@qq.com,dudu-2566@163.com,hxy_you@sina.com,lhm0210@ncpeu.edu.cn, zja@ncepu.edu.cn, \\ lsz@ncepu.edu.cn, ^lbx@ncepu.edu.cn
}

How to cite this paper: $\mathrm{Wu}, \mathrm{M} . \mathrm{Y} ., \mathrm{Xiong}$, J., Liao, L., Zhu, L., Zhang, R.X., Wu, Z., Du, G., Huang, X.Y., Li, H.M., Zhang, J., Liao, S.Z. and Lu, B.X. (2020) Nonlinear Switching Transient Field Simulation of Cable Joint without Residual Charge. Energy and Power Engineering, 12, 46-52.

https://doi.org/10.4236/epe.2020.124B005

Received: January 6, 2020

Accepted: April 7, 2020

Published: April 10, 2020

\begin{abstract}
The analysis of the impulse voltage on the internal electric field of the cable joint plays a key role in studying the breakdown of the joint. Based on the finite element method, a three-dimensional electromagnetic field simulation model of the cable joint is established in this paper. Simulation results show that the voltage at the head of the cable joint reaches about twice the impulse voltage. The increase of the conductivity of semi-conductive material also leads to the increase of electric field intensity. Then, several points and curves at different positions are selected for further analysis in this paper. Among them, the electric field distortion at the edge of the high voltage shield is the most serious and the electric field in the air gap is the least.
\end{abstract}

\section{Keywords}

Cable Joint, Switching Transient Field, 3D Transient Electromagnetic Simulation Model, Finite Element Method

\section{Introduction}

With the increasing use of power cables in medium voltage distribution networks, the number of cable joints in use is increasing. The quality of the cable joint manufacturing and installation varies, making the cable joint a weak link in medium voltage cables. Once the insulation performance of the cable joint is deteriorated, it will cause partial discharge and even cause serious accidents such as explosions, which will affect the safe operation of the power grid. During the switching operation, the overvoltage formed during the transient process prop- 
agates through the cable joints in the form of electromagnetic waves. Due to the existence of refraction and reflection phenomena, the components of each traveling wave are superimposed, which makes the transient overvoltage rises extremely fast, with large amplitude and high frequency, posing a great threat to the insulation of cable joints. When the conductivity of the semi-conductive material in the cable joint changes, it will affect the electric field distribution of the cable joint and seriously threaten the operational reliability of the cable joint [1].

In order to assure reliable operation of cable joint, a lot of research has been conducted on the space charge characteristics, interface breakdown and flashover characteristics, and optimization of the accessory structure, so as to minimize the possibility of problems in the production, installation and use of cable accessories [2] [3] [4] [5] [6]. These studies are based on steady-state fields and cannot reflect transient processes during switching operations. Therefore, in order to more accurately study the overvoltage and transient electromagnetic fields in cable joints, a full-wave simulation analysis of the three-dimensional electromagnetic field model is needed.

Based on the finite element method, a three-dimensional electromagnetic field simulation model of the cable joint is established in this paper. Under the shock wave form of double exponential wave, the electric field distribution and overvoltage in the cable joint are calculated, and the influence of the conductivity of the semi-conductive material on the electric field distribution and overvoltage is analyzed.

\section{Simulation Model Description}

\subsection{Structure and Material of Model}

The structure of the $10 \mathrm{kV}$ AC cable connector is shown in Figure 1. The model used in this paper is obtained by rotating the axisymmetric model around the axis of symmetry. The model consists of 9 parts: conductive copper, inner semi-conductive layer, main cable insulation layer, outer semi-conductive layer, stress cone, high-voltage shield, reinforced insulation layer and insulation shield. The material parameters of each part are shown in Table 1 .

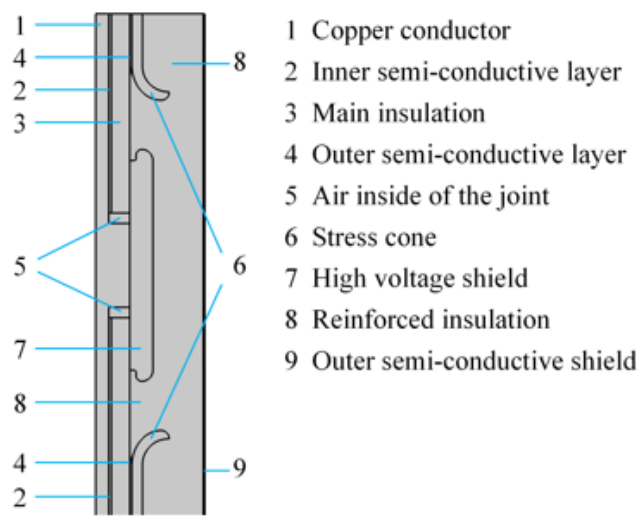

Figure 1. Structure of the $10 \mathrm{kV}$ cable joint. 


\subsection{Control Equation}

To calculate the transient field, Maxwell's equations need to be solved. In the region without charge density and current density, the existence of the potential $\varphi$ can be ignored, and only the magnetic vector potential $\boldsymbol{A}$ is used to represent the electric field strength and magnetic field strength. The control equation is shown in Equations (1) as follow.

$$
\nabla \times\left(\frac{1}{\mu_{\mathrm{r}}} \nabla \times \boldsymbol{A}\right)+\mu_{0} \sigma \frac{\partial \boldsymbol{A}}{\partial t}+\mu_{0} \varepsilon_{0} \frac{\partial}{\partial t}\left(\varepsilon_{\mathrm{r}} \frac{\partial A}{\partial t}\right)=0
$$

where $t$ is time, $\mu_{\mathrm{r}}$ is relative permeability, $\mu_{0}$ is the permeability of vacuum, $\sigma$ is electricity conductivity, $\varepsilon_{0}$ is the permittivity of vacuum, $\varepsilon_{\mathrm{r}}$ is relative permittivity.

The head and end of the cable joint are set as lumped port I and lumped port II, respectively, and the relationship between the voltage $U_{\text {port }}$ and current $I_{\text {port }}$ of the port is expressed as

$$
Z_{\text {port }}=\frac{U_{\text {port }}}{I_{\text {port }}}
$$

where $Z_{\text {port }}$ is the wave impedance of lumped port. The wave impedance of the lumped port at the head is set to $5 \Omega$. Although the internal impedance of the power supply should actually change with frequency, it is approximated in this paper with a small resistance. The impedance of the lumped port at the end is set to $5 \mathrm{G} \Omega$ to simulate an open circuit condition in the laboratory. And the curve of the overvoltage at the end of the cable joint is extracted from this port.

A wave excitation is applied at the head of the joint, and the waveform is shown in Figure 2. The equation of the impulse wave is expressed as

Table 1. Material parameter.

\begin{tabular}{cccc}
\hline Materials & Relative permittivity & Conductivity $(\mathrm{S} / \mathrm{m})$ & Application \\
\hline Copper & 1 & $5.8 \times 10^{8}$ & Cable conductor \\
XLPE & 2.3 & $1 \times 10^{-15}$ & Main insulation \\
Silicone Rubber & 4.3 & $2.727 \times 10^{-12} \times e^{9.796 \times 10^{-6} \times E}$ & Joint insulation \\
$\begin{array}{c}\text { Semi-conducting } \\
\text { material }\end{array}$ & 10 & $5,10,15,20$ & $\begin{array}{c}\text { Conductive layers and } \\
\text { stress cone }\end{array}$ \\
\hline
\end{tabular}

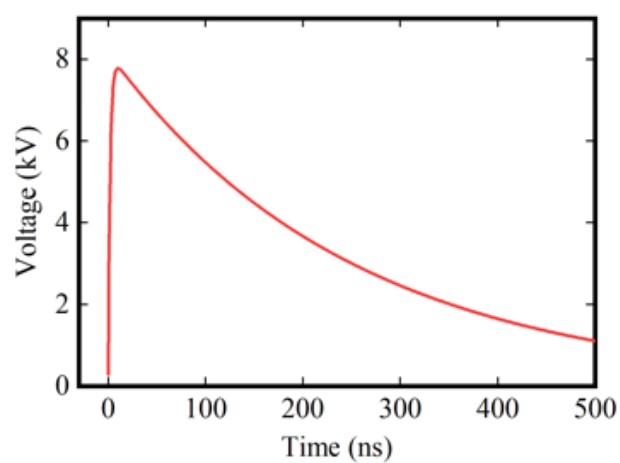

Figure 2. The waveform of the impulse wave. 


$$
U(t)=10 \sqrt{\frac{2}{3}}\left[\exp \left(-4 \cdot 10^{6} t\right)-\exp \left(-4.76 \cdot 10^{8} t\right)\right]
$$

Since the influence of residual charge is not considered, the initial condition of the magnetic vector potential $A$ is set as

$$
\begin{aligned}
& \boldsymbol{A}(\boldsymbol{r}, 0)=0 \\
& \left.\frac{\partial \boldsymbol{A}}{\partial t}\right|_{t=0}=0
\end{aligned}
$$

\section{Results and Discussion}

According to the methods and settings above, the overvoltage of lumped ports and the electric field distribution under different conductivity of semi-conductive materials are simulated.

\subsection{Simulation Analysis of Overvoltage under Different Conductivity}

The voltage of lumped port I and impulse voltage under different conductivity is compared in Figure 3. It can be seen from Figure 3(a) that the voltage of lumped port $\mathrm{I}$ is also in the form of a double exponential function, reaching twice the applied voltage. Figure 3(b) is enlarged by the voltage peak time of Figure 3(a). As can be seen from Figure 3(b), when the conductivity changes from $5 \mathrm{~S} / \mathrm{m}$ to $20 \mathrm{~S} / \mathrm{m}$, the voltage gradually decreases with a small change.

And the voltage of lumped port II under different conductivity is compared in Figure 4. Obviously, the voltage oscillation amplitude of lumped port II is larger, and the voltage decreases with the increase of conductivity.

\subsection{Analysis of Electric Field Distribution under Different Conductivity}

Figure 5 shows the distribution of the electric field under different conductivity when the voltage reaches its peak. As the conductivity increases, the maximum electric field intensity increases, but the growth rate slows down.

For a more detailed analysis of the distribution of the electric field, four points at different locations, namely $\mathrm{A}, \mathrm{B}, \mathrm{C}$ and $\mathrm{D}$, and four curves, $l_{1}, l_{2}, l_{3}$ and $l_{4}$, are

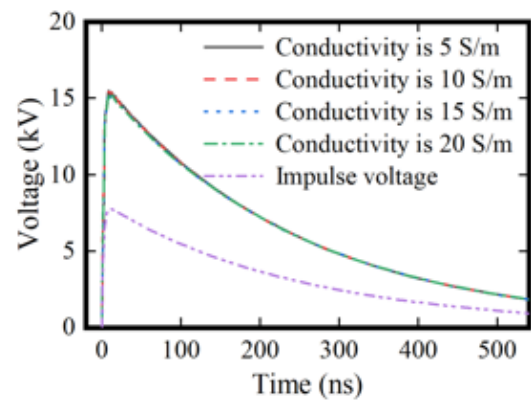

(a) global figure

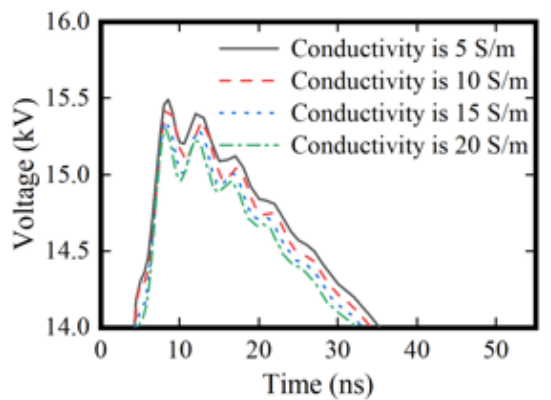

(b) zoomed curve

Figure 3. Voltage of the Lumped Port I vs. Time. 


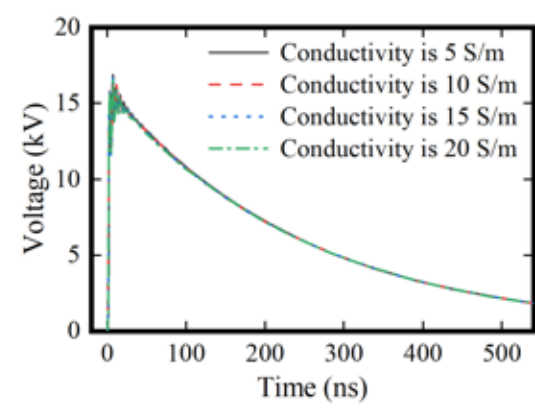

(a) global figure

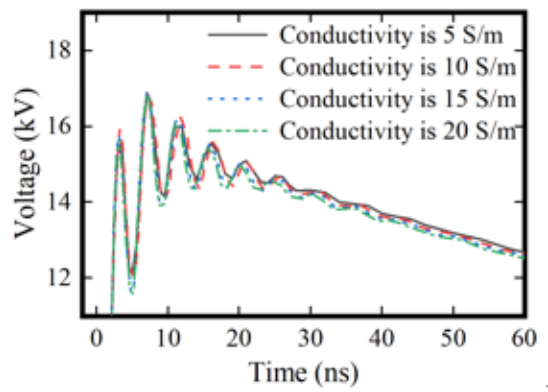

(b) zoomed curve

Figure 4. Voltage of the Lumped Port II vs. Time.

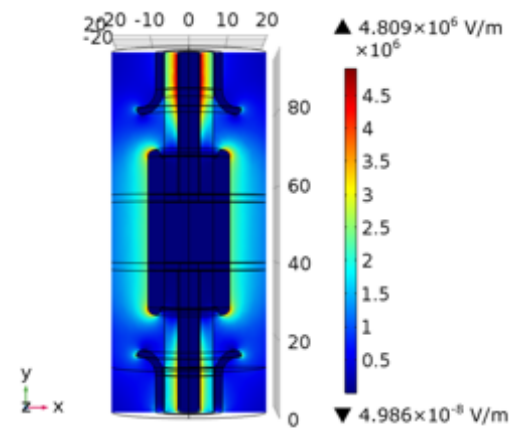

(a) Conductivity is $5 \mathrm{~S} / \mathrm{m}$

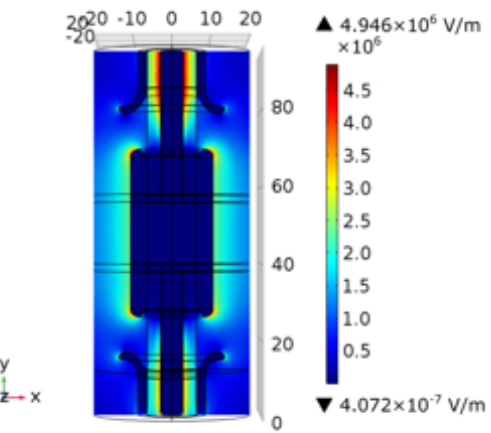

(c) Conductivity is $15 \mathrm{~S} / \mathrm{m}$

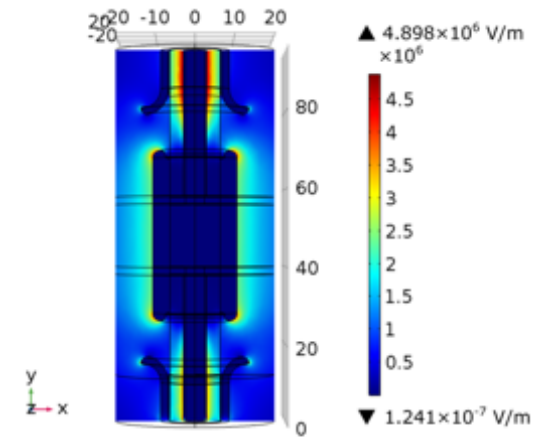

(b) Conductivity is $10 \mathrm{~S} / \mathrm{m}$

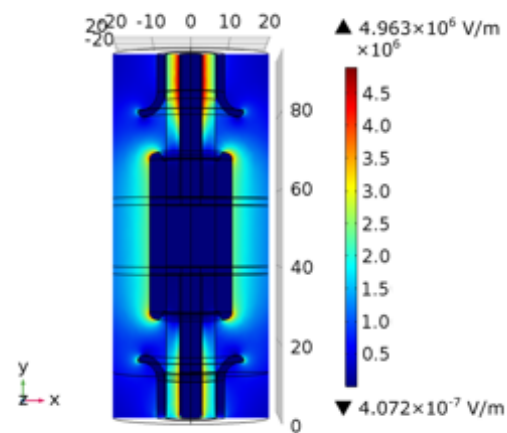

(d) Conductivity is $20 \mathrm{~S} / \mathrm{m}$

Figure 5. The distribution of the electric field under different conductivity.

selected, as shown in Figure 6.

Figure 7 shows the electric field intensity at point A, B, C and D under different conductivity over time. It can be seen that the conductivity has a greater effect on point $\mathrm{A}$ and $\mathrm{D}$, and a smaller effect on point $\mathrm{B}$ and $\mathrm{C}$. As the conductivity increases, the electric field intensity at point $\mathrm{A}$ increases. But the conductivity has the opposite effect on the electric field intensity at points $\mathrm{B}, \mathrm{C}$ and $\mathrm{D}$. Among the four points, the electric field distortion is the most serious at point $\mathrm{B}$.

Figure 8 shows the distribution of electric field intensity along the curve $I_{1}, l_{2}$, $I_{3}$ and $l_{4}$ when the voltage reaches its peak under different conductivity. As the conductivity increases, the growth rate of electric field intensity gradually saturates. Among the four curves, the electric field intensity on curves $I_{1}$ and $I_{2}$ is larger, while the electric field intensity on curves $l_{4}$ is the smallest. 


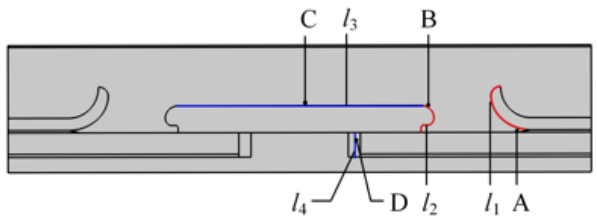

Figure 6. The distribution of the points and curves.

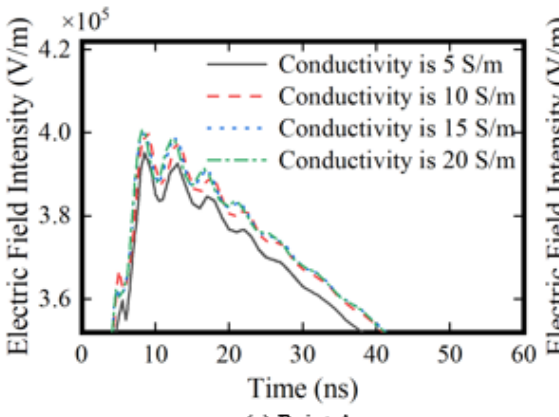

(a) Point $\mathrm{A}$

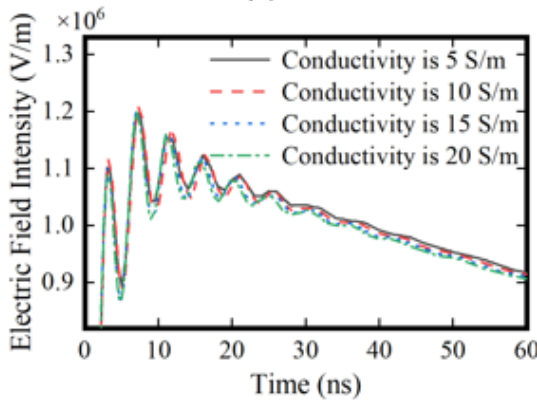

(c) Point C

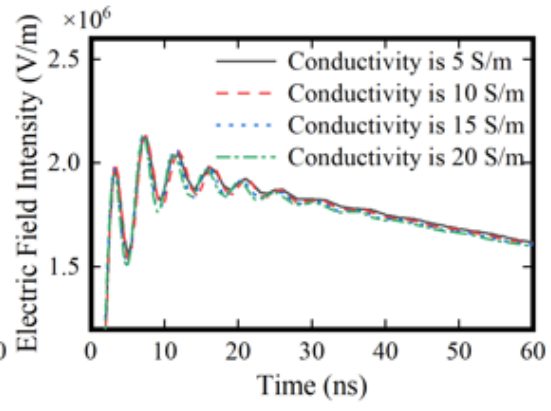

(b) Point B

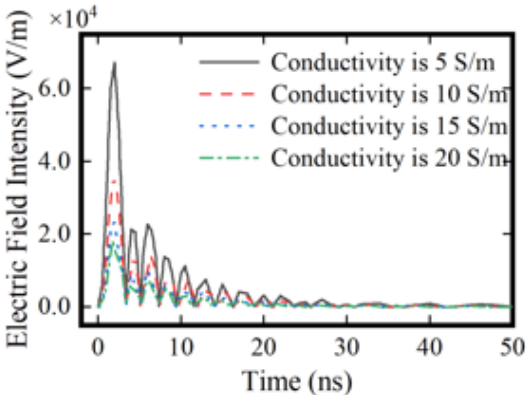

(d) Point D

Figure 7. The electric field intensity at point $\mathrm{A}, \mathrm{B}, \mathrm{C}$ and $\mathrm{D}$ under different conductivity.

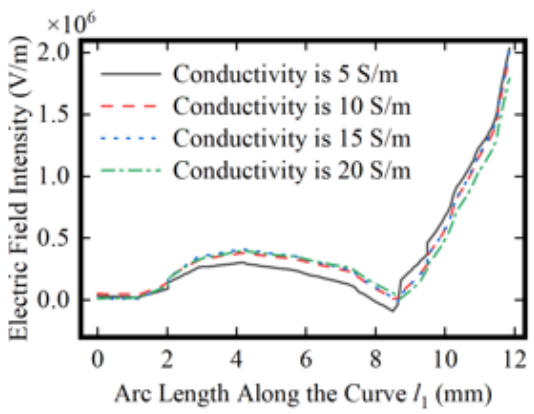

(a) Curve $l_{1}$

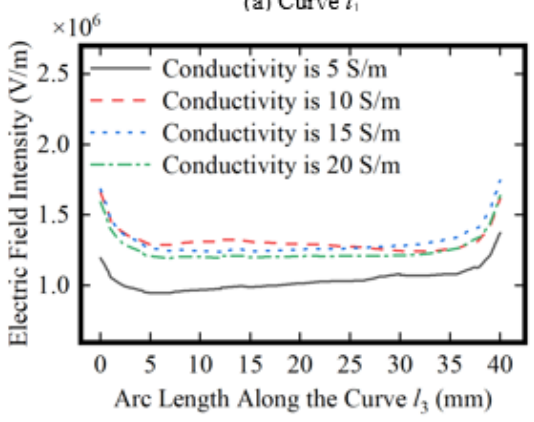

(c) Curve $l_{3}$

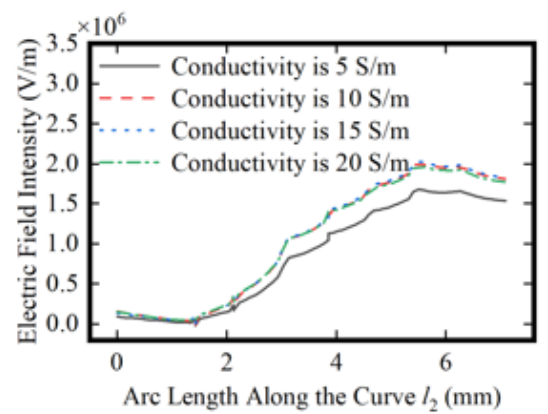

(b) Curve $l$,

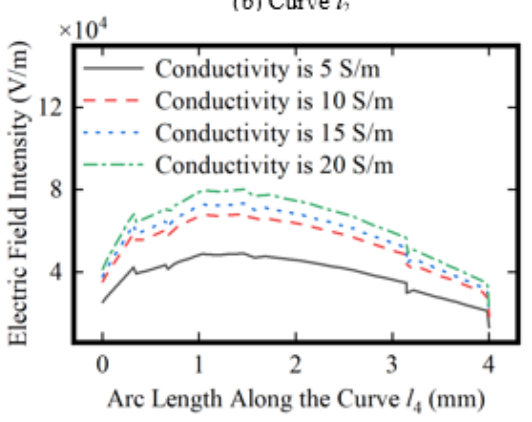

(d) Curve $l_{4}$

Figure 8. The electric field intensity along the curve $l_{1}, l_{2}, l_{3}$ and $l_{4}$ under different conductivity. 


\section{Conclusion}

Due to the multiple refraction and reflection inside the cable joint, the voltage at the head of the cable joint reaches about twice the impulse voltage. Moreover, the voltage oscillation amplitude at the end of the cable joint is larger than that at the head of the cable joint. As the conductivity increases, the voltage at the head and end decreases slightly. The increase of conductivity also leads to the increase of electric field intensity. After that, different points and curves at different positions are selected for further analysis in this paper. The electric field distortion at the edge of the high voltage shield is the most serious and the electric field in the air gap is the least.

\section{Acknowledgements}

This work was supported by the Science and Technology Project of China South Power Gird Co., Ltd., (GZHKJXM20180140).

\section{Conflicts of Interest}

The authors declare no conflicts of interest regarding the publication of this paper.

\section{References}

[1] Mazzanti, G., Montanari, G.C., and Palmieri, F. (2003) Quantities Extracted from Space-charge Measurements as Markers for Insulation Aging. IEEE Transactions on Dielectrics and Electrical Insulation, 10, 198-203.

http://dx.doi.org/10.1109/TDEI.2003.1194100

[2] Tohmine, T., Fujitomi, T., Miyake, H., Tanaka, Y., Ida, Y. and Inoue, Y. (2017) Measurement of Space Charge Accumulated in Multi-layered Samples Composed of Different Insulators Used in the Joints of DC Transmission Cables. 2017 International Symposium on Electrical Insulating Materials (ISEIM), Toyohashi, 2017, 299-302. http://dx.doi.org/10.23919/ISEIM.2017.8088746

[3] Yaroslavskiy, V., Walker, M., Katz, C. and Keefe, R. (2008) Comparative Laboratory Evaluation of Premolded Joints for Medium Voltage Cables. IEEE Transactions on Power Delivery, 23, 516-522. http://dx.doi.org/10.1109/TPWRD.2007.915909

[4] Aoran, X. and Yueping, M. (2019) Research Status and Trend of Insulation State Detection Technology for Power Cable Joints. Science and Technology Innovation and Application, 31, 28-31.

[5] Guo, Q., Xiong, J., Dai, N., Liao, S. and Lu, B. (2018) Influence of the Material Parameters on the Electric Field Intensity Distribution of $10 \mathrm{kV}$ Cable Elbow Connector. 2018 2nd IEEE Conference on Energy Internet and Energy System Integration (ED), Beijing, 2018, 1-5. http://dx.doi.org/10.1109/EI2.2018.8582320

[6] Heinrich, R., Bonisch, S., Pommerenke, D., Jobava, R. and Kalkner, W. (2000) Broadband Measurement of the Conductivity and the Permittivity of Semiconducting Materials in High Voltage XLPE Cables. 2000 Eighth International Conference on Dielectric Materials, Measurements and Applications (IEE Conf. Publ. No. 473), Edinburgh, 2000, 212-217. http://dx.doi.org/10.1049/cp:20000507 